\title{
Recent Developments in ADC Technology: Preclinical Studies Signal Future Clinical Trends
}

\author{
Penelope M. Drake ${ }^{1}$ David Rabuka ${ }^{1}$
}

Published online: 8 November 2017

(c) The Author(s) 2017. This article is an open access publication

\begin{abstract}
The antibody-drug conjugate (ADC) field is in a transitional period. Older approaches to conjugate composition and dosing regimens still dominate the ADC clinical pipeline, but preclinical work is driving a rapid evolution in how we strategize to improve efficacy and reduce toxicity towards better therapeutic outcomes. These advances are largely based upon a body of investigational studies that together offer a deeper understanding of the absorption, distribution, metabolism, and excretion (ADME) and drug metabolism and pharmacokinetics (DMPK) fates of both the intact conjugate and its smallmolecule component. Knowing where the drug goes and how it is processed allows mechanistic connections to be drawn with commonly observed clinical toxicities. The field is also starting to consider ADC interactions with the immune system and potential synergistic therapeutic opportunities therein. In an indication of future directions for the field, antibody conjugates bearing non-cytotoxic small-molecule payloads are being developed to reduce side effects associated with treatment of chronic diseases. ADCs are not a magic bullet to cure disease. However, they will increasingly become valuable therapeutic tools to improve patient outcomes across a variety of indications.
\end{abstract}

David Rabuka

david.rabuka@catalent.com

1 Catalent Biologics, 5703 Hollis Street, Emeryville, CA 94608, USA

\section{Key Points}

The clinical pipeline of antibody-drug conjugates (ADCs) has dramatically changed since 2013 , as the rapid technological advances made in the preclinical space during the early part of the decade have begun to mature into clinical-stage projects.

Iterative but meaningful improvements in ADC technology will continue as the clinical-preclinicalclinical feedback loop focuses heavily on approaches for reducing off-target toxicities and improving patient outcomes through changes in both ADC composition and clinical trial study design.

Another major focus in both clinical and preclinical arenas will be investigating combination therapies, with particular attention on how ADCs can complement immunooncology approaches. Expanded use of ADCs beyond oncology indications is also anticipated.

\section{Introduction}

Antibody-drug conjugates (ADCs) are a class of drugs characterized by an antibody scaffold covalently modified with a variable number of small-molecule payloads. These drugs are hybrid entities combining both biologic and small-molecule characteristics, where the antibody serves to target the small molecule specifically to the intended cell type. Typically envisioned as oncology therapeutics, ADCs represent an old concept [1] that has proven difficult to translate into clinical successes. Current ADC technology 
relies on selecting an appropriate target antigen, one that is selectively expressed on tumor cells and internalizes upon antibody engagement. ADCs comprise three parts-the antibody, the small-molecule payload, and the linker joining them together. A critical quality attribute of any $\mathrm{ADC}$ is the amount of drug loading, or the average ratio of conjugated payload to antibody. This is referred to as the drug-to-antibody ratio (DAR). Since the DAR dictates the amount of payload delivered per internalized antibody, it strongly influences both efficacy and toxicity. In addition, depending on the conjugation and linker technologies used, high-DAR ADCs can have poor biophysical characteristics (e.g., hydrophobicity, aggregation) that reduce efficacy and increase toxicity. Certain conjugation and linker technologies can mitigate these effects and yield better-behaved high-DAR species [2].

The features that make for a good ADC antibody are generally the same features that are desirable in any modern therapeutic antibody [3] and are not covered in this review. To date, the majority of clinically-tested ADC payloads are either antimitotic/microtubule inhibiting (e.g., auristatins, maytansinoids, tubulysin) or DNA alkylating (e.g., pyrrolobenzodiazepines, indolinobenzodiazepines, calicheamicins, duocarmycins), although a handful of other interesting payloads with novel mechanisms of action have been introduced (e.g., irinotecan derivatives and $\alpha$-amanitin). The antimitotic and DNA alkylating compound classes tend to have in vitro $50 \%$ inhibitory concentration (IC50) potency values in the $\mathrm{nM}$ and $\mathrm{pM}$ ranges, respectively. These differences in potency are also reflected in the clinically-achievable maximum tolerated doses for the two payload classes [4].

Linkers are the most modifiable aspect of an ADC; changes made to linkers can impact the biophysical and functional performance of the entire conjugate for good or bad. Linkers are attached to antibodies using a variety of conjugation approaches that fall into two general categories: those resulting in heterogeneous or in site-specific payload placement. The former tend to exploit the reactivity of endogenous lysine or hinge cysteine residues within the antibody, whereas the latter use reactive chemical handles exogenously introduced into particular locations on the antibody through various means. While the field is coming to appreciate the functional and analytical advantages of employing site-specific conjugation [2], the ADC clinical pipeline is still dominated by heterogeneous conjugates. ADC linkers can be either noncleavable, designed to persist through antibody degradation in the lysosome, or cleavable, designed to be stable in the circulation but readily degraded after internalization by the target cell. The relative stability of various linkers and conjugation chemistries can have a major effect on both efficacy and toxicity [2].

\section{The Changing Clinical ADC Landscape}

The landscape of ADC therapeutics is changing rapidly. With two new approvals in 2017, the number of ADCs authorized for use by the US FDA has doubled. The recently approved drugs are inotuzumab ozogamicin (Besponsa) and gemtuzumab ozogamicin (Mylotarg); both are calicheamicin conjugates from Pfizer and target cluster of differentiation (CD)-22 and CD33, respectively, on liquid tumors. Besponsa was approved for treatment of relapsed/ refractory acute lymphoblastic leukemia (ALL) $[5,6]$. Mylotarg, the very first marketed ADC, was originally approved in 2000 for treatment of CD33-positive acute myeloid leukemia (AML). However, it was withdrawn from the market in 2010 due to treatment-related toxicity concerns. Mylotarg won reapproval in 2017 with a lower recommended dose and altered dosing schedule. These two drugs join approved ADCs Adcetris, an anti-CD30 monomethyl auristatin $\mathrm{E}$ (MMAE) conjugate marketed to treat relapsed/refractory Hodgkin lymphoma and systemic anaplastic large cell lymphoma, and Kadcyla, an antiHER2 DM1 conjugate used to treat HER2+ metastatic breast cancer. The latter drug is currently the only FDAapproved ADC for treatment of solid tumors.

With four approved drugs in the class, ADCs are beginning to realize some of the promise they have long offered. A renaissance in the sector began in the past decade, led by companies such as Immunogen and Seattle Genetics that offered conjugation and linker/payload technology platforms enabling pharmaceutical companies to rapidly test ADCs against a number of targets. Adcetris and Kadcyla were developed during this time, with FDA approvals in 2011 and 2013, respectively. In early 2013, there were about 20 ADCs in the clinic and nearly $80 \%$ of those were conjugated to antimitotic drugs, namely auristatins (e.g., MMAE and monomethyl auristatin F [MMAF]) or maytansinoids (e.g., DM1 or DM4). The conjugates were heterogeneous mixtures that relied on the chemical reactivity of lysine or hinge cysteine residues in order to attach linker/payloads.

The number and makeup of ADCs in the clinical pipeline has changed as the field matures. The first site-specific ADC to reach the clinic, vadastuximab talirine, is an antiCD33 antibody conjugated through engineered cysteine residues in the heavy chain to yield a DAR 2 molecule. This ADC, which began phase I trials in mid-2013 was also notable as the first clinical ADC to bear a pyrrolobenzodiazepine (PBD) payload, a highly potent DNA alkylator. From 2013 to 2017, the number of ADCs in clinical trials more than tripled [7], and site-specific ADCs made up nearly $15 \%$ of that total. Meanwhile, the share of antimitotic payloads dropped from 80 to $<65 \%$ overall, and 
accounted for only one-third of site-specific ADCs. In general, the trend away from antimitotic payloads reflects a move towards more potent cytotoxic drugs, particularly DNA alkylators, in order to access a broader range of target antigens that may be expressed at lower cell surface levels or may require alternate mechanisms of action beyond microtubule inhibition.

In part, the shift to more potent warheads is a response to lackluster clinical results achieved with ADCs bearing antimitotic payloads. According to a recent review by Beck et al. [7], nearly $40 \%$ of ADCs bearing maytansine, MMAE, or MMAF that entered clinical trials were later discontinued, presumably due to lack of efficacy or (rarely) excessive toxicity. In a few cases, the same target antigen (e.g., CD70 or CD19) was repurposed for a second clinical attempt with a new ADC carrying a more potent payload with a different mechanism of action. However, highly potent payloads carry an increased safety risk to patients. The recent termination of the phase III trial for vadastuximab talirine due to toxicity concerns [8], even while the drug conferred a 70\% complete remission rate for AML patients [9], highlights that the balance of potency and safety must be carefully tuned and that scientists and regulators are still working to get this right.

As the field comes to grips with these clinical challenges, the landmark preclinical studies of the past 2 years have tended towards methodical explorations focused on specific problem areas within the field. The remainder of this review focuses on papers published between 2015 and 2017 that we believe address important questions and provide insight into where the field is headed. We refer readers to three other excellent ADC reviews-from Beck et al. [7], Polakis [10], and Govindan et al. [4] — to gain a comprehensive sense of the state of the art.

\section{Preclinical Studies Addressing Clinically- Relevant Toxicity Issues}

A particular emphasis has been placed on understanding mechanisms of ADC toxicity, in large part because ADCs of the 2013 era-heterogeneous conjugates bearing antimitotic payloads-exhibited prominent off-target effects, such that the dose-limiting toxicities were typically observed in nontarget-expressing tissues. In 2015, Saber and Leighton [11] published a systematic review of toxicity studies submitted in 20 investigational new drug (IND) applications and concluded that ADC toxicity was not driven by target antigen but rather by linker/payload. ADCs sharing the same linker/payload composition tended to reach the same maximum tolerated dose, even when their target antigens showed endogenous expression in completely different tissue/organ compartments. This sobering observation revealed how far the field still has to go in terms of achieving specific cytotoxic payload delivery to tumor cells while sparing healthy tissues. But it also offers a possible explanation for the high failure rate of 2013-era ADCs. It is likely that the lack of clinical benefit observed for some ADCs was the result of an inability to dose to an efficacious level due to off-target toxicities driven by the linker/payload. Indeed, de Goeij and Lambert [12] compared dosing requirements to achieve preclinical efficacy in mouse tumor models with clinically achievable doses for a number of ADCs and concluded that, in many cases, the clinical dose was insufficient for the ADC to reach its maximal potential effect.

\subsection{An Investigation of a Dose-Limiting Toxicity for MMAE Conjugates}

If ADC off-target toxicity can be controlled, then the maximum tolerated dose can likely be increased, perhaps leading to better clinical response to treatment. Neutropenia is a common, often dose-limiting, toxicity noted in patients treated with ADCs conjugated to MMAE through a linker containing the protease cleavable dipeptide ValCit (vc) $[13,14]$. By contrast, patients treated with ADCs conjugated to the similar auristatin payload, MMAF, through a noncleavable maleimidocaproyl (mc) linker, do not typically experience neutropenia. From these observations, it has been thought that proteolytic payload release mediates the toxicity, however the details have not been understood. Considering that nearly $30 \%$ of clinically-used ADCs carry a vc-MMAE linker/payload, and that fully $50 \%$ of ADCs in the clinic contain a cleavable dipeptidebased linker, understanding the mechanisms driving this toxicity could impact many therapeutic programs [7]. To this end, researchers from Agensys differentiated neutrophils in vitro and assessed the cells' sensitivity to ADC treatment at different stages of maturation [15]. Fullymature neutrophils were not affected by incubation with ADCs. By contrast, differentiating neutrophils were sensitive to treatment with vc-MMAE-conjugated ADCs but much less sensitive to mc-MMAF-conjugated ADCs. Further experiments showed that the developing neutrophils secreted the serine protease elastase, which could cleave the vc-MMAE linker and release free payload. The authors also investigated the role of $\mathrm{Fc}$ gamma receptor $(\mathrm{Fc} \gamma \mathrm{R})$ mediated ADC internalization in neutrophil cytotoxicity. While this pathway was not required for sensitivity to vcMMAE-conjugated ADCs, the modest sensitivity of differentiating neutrophils to mc-MMAF-conjugated ADCs could be attributed to this nonspecific interaction of ADC 
Fc constant region with $\mathrm{Fc} \gamma \mathrm{Rs}$ expressed on developing neutrophils. Fc $\gamma$ R-mediated internalization has also been shown to play a role in ADC inhibition of platelet development in vitro [16], providing a possible explanation for the dose-limiting toxicity of thrombocytopenia that is associated with certain ADC treatments, including Kadcyla $[5,17]$.

\subsection{An Update on ADC-Related Liver Toxicity}

ADCs - particularly maytansine conjugates, such as Kadcyla-also commonly induce hepatic toxicity in patients. A consensus is building in the field that overly conjugated (DAR $\geq 8$ ) ADCs are rapidly cleared by Kupffer cells in the liver. Papers from both Seattle Genetics and ImmunoGen demonstrated this phenomenon and showed that liver uptake of highly conjugated ADCs began within the first 15-30 min after dosing [7, 18, 19]. These observations highlight the need for manufacturing controls to regulate DAR distribution. The most effective means to this end is through the use of site-specific conjugation technologies, and this is one reason the field has begun moving rapidly in that direction. Notably, the effect of a high DAR can be mitigated by using a sufficiently hydrophilic linker $[4,7,10,19]$, demonstrating the relationship between hydrophobicity and liver-mediated clearance and underscoring the utility of using linker chemistry to control ADC biophysical and functional characteristics.

Conjugation chemistry matters too. A commonly used reactive chemical handle-maleimide, which reacts with exposed thiol groups on free cysteine residues-can be unstable in vivo and undergo a retro-Michael reaction, resulting in the transfer of linker/payload to cysteine on another protein carrier, such as albumin. When a maleimide-conjugated vc-MMAE-bearing ADC is dosed in vivo, the retro-Michael reaction can result in formation of an albumin-vc-MMAE conjugate. This phenomenon had been observed previously [20, 21], but a group from Pfizer quantified it for the first time in 2017 [22]. At $48 \mathrm{~h}$ post-dose, albumin-vc-MMAE represented $4 \%$ of total circulating payload and $\sim 35 \%$ of all MMAE that was deconjugated from the antibody [22]. Slowing or stopping this linker/payload transfer to albumin represents a good opportunity for mitigating offtarget toxicities, as the distribution and disposition profiles of albumin conjugates will likely differ from those of antibody conjugates. Chemistries designed to open the maleimide ring after ADC production can prevent the retro-Michael reaction from occurring and avoid these complications [7, 23, 24]. However, the extent to which these stabilizing treatments have been applied to ADCs in the clinic is unclear.

\subsection{Toxicity Considerations for ADCs Bearing Highly Potent DNA Alkylating Payloads}

The broadest clinical ADC experience to date has been with conjugates carrying antimitotic payloads. As referenced in the preceding sections, such ADCs tend to show prominent organ toxicities in the hematopoietic compartments and in the liver. Much less is known about the toxicities that will be associated with the newer classes of DNA alkylating payloads, such as the PBDs. The two PBD conjugates that have advanced the furthest in the clinic, vadastuximab talirine (targeting CD33) and rovalpituzumab tesirine (targeting DLL3), are both conjugated via protease cleavable linkers and have both commonly shown hematopoietic dose-limiting toxicities in clinical trials $[15,25,26]$. Similarly, the recently approved calicheamicin ADC conjugates, Besponsa and Mylotarg, which represent another class of potent DNA alkylating payloads, conjugated via hindered disulfide cleavable linkers, have exhibited prominent hematopoietic doselimiting toxicities $[5,16,27]$.

These data points notwithstanding, there is much less accumulated knowledge about the clinical effects of dosing DNA alkylators. Therefore, preclinical studies can help to bridge our understanding of the in vivo consequences of dosing with an ADC bearing a DNA alkylating payload. In a recent preclinical study from Genentech, Ma et al. [28] assessed the percent of PBD payload that was covalently bound to DNA after a single $5 \mathrm{mg} / \mathrm{kg}$ intravenous bolus dose of an anti-CD22 PBD ADC in a mouse non-Hodgkin lymphoma (NHL) xenograft model. Drug distribution in tumor, liver, lung, and kidney was monitored at 24 and $96 \mathrm{~h}$ post-dose. The authors found that $>98 \%$ of PBD recovered from tumor, and $\geq 78 \%$ of PBD recovered from liver or lung, was bound to DNA, and concluded that DNA binding should be considered a disposition pathway for this payload. Gratifyingly, the enrichment of DNA-bound PBD in tumors as compared to liver or lung was $\sim 40$-fold at $96 \mathrm{~h}$ post-dose. Very little bound PBD was detected in the kidney. Considering that the effects of DNA-bound payload in healthy tissues could be cumulative unless abrogated via DNA repair mechanisms, it would be informative to see the results of a similar multidose study to monitor PBD accumulation or clearance from healthy tissues.

In this vein, an interesting study by Hinrichs et al. [29] described the potential tolerability benefits of dose fractionation for ADCs bearing PBD payloads. First, the authors demonstrated, by using two ADCs tested in five xenograft models, that in vivo ADC efficacy was similar when the same amount of ADC was delivered either as a single dose or as three fractionated weekly doses. Next, the authors used a similar dosing schedule to treat rats or cynomolgus monkeys with (different) ADCs bearing PBD 
payloads. In both species, animals receiving three fractionated weekly doses fared better than animals receiving a single bolus dose. For example, the rats showed improvements in survival, body weight, and hematological readouts, whereas the monkeys showed improvements in body weight loss and less severe renal injury. Finally, pharmacokinetic studies in both rats and monkeys demonstrated that the overall area under the concentration-time curve $\left(\mathrm{AUC}_{0-28}\right)$ during the 28-day study period was comparable for both dosing schedules. By contrast, the peak plasma concentration $\left(C_{\max }\right)$ was approximately threefold higher in animals receiving the single dose than in those receiving the fractionated doses. Accordingly, Hinrichs et al. [29] concluded that efficacy is driven by overall ADC exposure, whereas $C_{\max }$ dictates toxicity.

\section{Designing a Better Preclinical Efficacy Study}

\subsection{Improving ADC Tumor Penetration}

Another important study regarding ADC dosing and efficacy seems to contradict some of the conclusions that lead from the Hinrichs et al. [29] work. In a 2016 article, Cilliers et al. [30] began with a physiologically-based pharmacokinetic (PBPK) model of systemic ADC distribution, proceeded to proof-of-concept in vivo studies to test the model, and ended with a literature survey citing examples of published studies that supported their model. This thoughtful work focused on ADC biodistribution in the tumor and in healthy tissues, particularly the relationship of ADC tumor penetration to ADC (or unconjugated antibody + ADC) dose. Namely, the authors showed that when mice were given a dose of trastuzumab-DM1 (TDM1; Kadcyla) at $3.6 \mathrm{mg} / \mathrm{kg}$ (the clinical dose), ADC distribution was highly heterogeneous within the tumor, showing predominant localization next to blood vessels. When unconjugated trastuzumab (the antibody used to make the T-DM1 conjugate) was co-dosed along with the clinical dose of T-DM1, the ADC was able to penetrate deeper into the tumor (presumably due to competition for antigen binding near the site of extravasation), and the distribution became more homogenous [30]. Meanwhile, including the unconjugated antibody had little effect on T-DM1 accumulation in healthy tissues. To assess the potential impact of their observations on ADC efficacy (which was not included in their study), the authors surveyed papers from the literature where ADCs with the same linker/payload but different DARs were dosed such that the amount of payload delivered was held constant but the amount of antibody varied. The general trend in these studies was that dosing with more antibody resulted in improved efficacy [30]. The authors suggested that this improvement may have been due to better ADC tumor penetration. If true, then their work has implications for preclinical, and perhaps clinical, study design. It may point the way towards improving efficacy outcomes without dosing more drug - thus widening the therapeutic window.

How can these conclusions be reconciled with those from the study by Hinrichs et al. [29], which showed that higher ADC dosing had no effect on efficacy? We need to look closer at the details of each study. Certain ADC attributes affected the PBPK biodistribution model predictions and-in some cases (e.g., a few of the literature studies) - the observed real-world outcomes. The critical inputs included payload potency (IC50), overall ADC clearance rates, target antigen cell surface copy number, and time post-dose (with tumor penetration becoming more homogenous over time). Considering that many of these critical inputs differed between the Hinrichs et al. [29] and Cilliers et al. [30] analyses, then perhaps a deeper understanding of how those differences can impact preclinical ADC efficacy would harmonize the apparent discordance between these two fine studies.

\subsection{Contributions of the Innate Immune System to ADC Efficacy}

Many groups are now beginning to consider the potential of the adaptive immune system to augment or complement the in vivo efficacy of their ADCs, particularly with respect to testing combination therapies of ADCs dosed along with checkpoint inhibitor drugs [31]. The possibilities for synergy are good given that many ADC payloads induce immunogenic cell death in their targets [32-34]. However, few groups have discussed the role that the innate immune system might already be playing in the results of routine mouse xenograft studies carried out in immunocompromised mice. No longer, since, Li et al. [35] from Seattle Genetics demonstrated that tumor-associated macrophages (TAMs) could be found infiltrating a number of cell linederived xenograft (CDX) models established in NOD scid gamma (NSG) and severe combined immunodeficiency (SCID) mice, and that the presence of these immune cells correlated with anti-tumor activity exhibited by isotype control ADCs. The authors went on to show that the TAMs mediated anti-tumor efficacy through Fc $\gamma \mathrm{R}$-based ADC internalization and processing, followed by the release of free payload into the tumor microenvironment. Consistent with this bystander killing mechanism, only membranepermeable payloads (such as MMAE) but not membraneimpermeable payloads (such as MMAF) could mediate the isotype-based tumor growth inhibition. These data suggest that additional controls may be needed in preclinical xenograft studies in order to fully understand the source of any observed efficacy. Encouragingly, a similar innate 
immune-based mechanism may also be at work in patients. The authors cite several examples of clinically-tested ADCs where clinical response was uncoupled from target antigen expression [36-38]. If these examples indeed represent TAM-mediated bystander killing of patient tumor cells, then perhaps a study designed to investigate this relationship might rapidly uncover new patient cohorts that could benefit from existing medicines.

\subsection{Designing a Better Study: Combination Therapies}

A more strategic approach to study design also means exploiting opportunities for drug synergy; specifically, finding drug combinations where the mechanisms of action intersect with the tumor biology so as to improve efficacy. Two recent studies from Immunomedics illustrate how this approach can work. In the first, Chang et al. [39] used preclinical studies to support a rationale for combination dosing to overcome ADC drug resistance due to tumor upregulation of multidrug resistance (MDR) efflux transporters. Namely, the authors proposed co-dosing the ADC along with small-molecule drugs that inhibit MDR efflux activity. In the second study, Cardillo et al. [40] explored the opportunities available from co-dosing an ADC bearing the $\mathrm{SN}-38$ payload (a topoisomerase I inhibitor) along with a poly(ADP-ribose) polymerase inhibitor (PARPi) that is also in clinical development. The PARPi disrupts DNA repair mechanisms, whereas $\mathrm{SN}-38$ promotes DNA strand breaks. When dosed together, the drugs achieved synergic efficacy due to their combined DNA-damaging effects, resulting in greatly improved tumor xenograft control as compared to that attained with the individual monotherapies. Together, these promising results lead us to hope that the field will see more rationally-designed opportunistic combination studies in the near future.

\section{Building a Better ADC}

It remains true that the most direct way to alter the efficacy and toxicity of an ADC is to change the composition of the molecule itself. Our final section describes recent progress towards this end.

\subsection{Strategies in ADC Target Antigen Selection}

A critical ADC feature that has implications for both efficacy and toxicity is the choice of target antigen. The cancer stem cell paradigm holds that a subpopulation of tumor cells (cancer stem cells or tumor-initiating cells) propagates disease, and that effective cancer treatment should eliminate them. Various markers exist for cancer stem cell or tumor-initiating cell (TIC) identification, and two have been selected as ADC target antigens. The target that has advanced the furthest is delta-like protein 3 (DLL3), recognized by the ADC rovalpituzumab tesirine. This drug, a PBD conjugate, is currently being tested in phase III clinical trials for the treatment of small-cell lung carcinoma. Results reported from a phase I study showed an $18 \%$ objective response rate (ORR), with a $38 \%$ ORR in patients with high DLL3 expression levels [26]. The second tumor-initiating cell antigen being tested as an ADC target is protein tyrosine kinase 7 (PTK7), expressed on TICs isolated from patient-derived tumor xenografts (PDX) representing several solid tumor types. Preclinical work from Pfizer and Abbvie/Stemcentrx describes an anti-PTK7 ADC conjugated through a ValCit dipeptide linker to an auristatin payload [41]. The ADC caused tumor growth inhibition in several PDX tumor models. Interestingly, through in vivo serial passage of tumor cells remaining after ADC treatment, the authors demonstrated that the drug also reduced the frequency of TICs in tumor tissue over time.

Another approach to controlling tumors is to limit their blood supply by targeting tumor vasculature. Seaman et al. [42] described ADCs against CD276, an antigen that is expressed on both tumor cells and tumor endothelial cells (in some cancers) but not on endothelium in healthy tissues. The authors hypothesized that simultaneously eliminating both of these populations within the tumor environment would yield greater overall tumor control. In preclinical studies, they generated two anti-CD276 ADCs that used the same antibody but carried different payloads, either MMAE or a PBD [42]. While both ADCs exhibited tumor growth inhibition against CD276-expressing xenograft models, only the PBD-conjugate was effective in vitro against tumor endothelial cells. In accord with the authors' hypothesis, the PBD ADC, which could simultaneously kill both tumor compartments (tumor cells and tumor endothelium), yielded better overall in vivo tumor control and more enduring complete responses as compared to the MMAE conjugate.

It has been appreciated for some time that internalization and subsequent trafficking through endosomal and lysosomal compartments does not proceed equally for all target antigens. An early study that highlighted this point was work by Polson et al. [43], which studied the in vivo efficacy of ADCs against seven NHL target antigens. The authors showed that ADCs bearing cleavable linkers could mediate tumor growth inhibition against all seven targets, whereas ADCs bearing noncleavable linkers were only efficacious against two of the seven targets. These varied target antigen requirements for cleavable versus noncleavable linkers suggested that the internalization/trafficking component of ADC processing plays a critical role 
in its function. Because cleavable linkers are susceptible to conditions found in endosomal compartments (i.e., prelysosome), the trafficking requirements for liberation of the attached payloads appear to be less strict than those for ADCs carrying noncleavable linkers, which are thought to require complete lysosomal degradation before payload release. However, the mechanistic relationship between internalization, intracellular trafficking, and ADC potency remains poorly understood [44]. A recent study offers a potential new approach for improving the trafficking characteristics of ADC target antigens.

DeVay et al. [45] took advantage of bispecific technology to produce an ADC targeting both a tumor-specific antigen (HER2) and a cell surface antigen that efficiently traffics to the lysosome upon engagement (amyloid precursor-like protein 2 [APLP2]). The idea of hitching a ride to the lysosome on the back of a convenient target is creative, and HER2 is a good model system for testing the hypothesis because it is a very well-studied ADC target that internalizes poorly and is thought to recycle back to the cell surface after internalization [46]. The authors found that their dual targeting approach did result in improved in vitro potency against HER2+ cell lines. However, the in vivo efficacy of the bispecific ADC was worse than that of the conventional (monospecific but bivalent) anti-HER2 ADC. The authors postulated that this result reflected the importance of the bivalent anti-HER2 interaction for in vivo activity. Future iterations of this approach could maintain the bivalency of the targeting antibody component while also incorporating the internalization component.

\subsection{Recent Advances in Linker Technology}

The linker of an ADC simultaneously serves several purposes. An important one is to physically join the antibody and small-molecule payload with a stable linkage that persists during ADC circulation in the bloodstream. Furthermore, the chemical ligation of linker to payload must be achieved without compromising biological potency. In this regard, the payload's structure (and any known structure-activity relationship [SAR] information) will dictate which reactive chemical groups may be used for ligation. Primary and secondary amines are most commonly accessed. Reactions using alternate functional groups are rare, either because they are difficult to achieve or because the resulting products are unstable. Two interesting papers from 2016 described significant progress towards broader functional group accessibility. Staben et al. [47] developed a chemoselective ligation approach that targets tertiary and heteroaryl amines coupled through a so-called "traceless" cleavable linker. After proteolytic cleavage of the linker, an adjacent self-immolating unit cyclizes upon itself and releases the payload in an unmodified form. An additional benefit of the ligation approach is that the quaternary amine produced from the reaction offers added solubility to the overall construct. This is a very desirable feature and is particularly useful when ligating hydrophobic payloads, such as those often found in ADCs. Kolakowski et al. [48] created a self-immolating linker (methylene alkoxy carbamate $[\mathrm{MAC}]$ ) that can attach to alcoholic payloads. The authors coupled the MAC unit with a $\beta$-glucuronidasepromoted release system, allowing for payload liberation upon ADC internalization. ADCs made using this linker system were stable over 7 days in mouse plasma and were highly efficacious in a mouse xenograft model.

Payloads that lose biological potency when the core chemical structure is modified require the use of traceless linkers. These systems comprise a cleavage event (the trigger) followed by the self-immolation event that releases the free payload. The kinetics of both cleavage and immolation can vary according to the structure of the linker/payload. These features are often tunable and are a common focus of linker/payload SAR investigations. A series of studies from Genentech addressed these considerations in the context of PBD payloads. The authors performed in vitro studies designed to induce cleavage/ immolation of various linkers and monitored the results by liquid chromatography/mass spectrometry (LC/MS) [49]. The data identified linkers that released free payload and found a group of linkers that cleaved but failed to selfimmolate, leaving a portion of the linker attached to the payload. The latter constructs were not potent in vitro or in vivo [50].

For payloads that tolerate chemical elaboration, noncleavable linkers afford an opportunity to adjust payload functionality. In this vein, Singh et al. [51] introduced a triglycyl peptide linker designed to overcome many biological limitations currently imposed on the efficacy of noncleavable conjugates. Specifically, the authors sought to (1) limit the extent of lysosomal proteolysis required for payload liberation, (2) improve payload transit from the lysosome into the cytosol, and (3) hinder payload transit from the extracellular space into neighboring cells. The triglycyl linker project deserves recognition for addressing these limitations. The results of their studies show that ADCs made using the triglycyl linker with a maytansine payload (DM1) were more efficacious against some cell lines and xenograft models as compared to ADCs made using the $N$-succinimidyl 4-(maleimidomethyl)cyclohexanecarboxylate (SMCC)-DM1 linker/payload. Against other cell lines and xenograft models, ADCs made using the two linker types showed comparable efficacy. Notably, use of the triglycyl design effectively turned the linker into a cleavable, but not traceless, system. Considering that the endosomal/lysosomal trafficking requirements are less 
stringent for ADCs with cleavable as compared to noncleavable linkers (a feature that the authors hoped to exploit), it is difficult to assess how much of the improved efficacy resulted from improved lysosomal escape based on linker charge at low $\mathrm{pH}$. However, it would be very interesting to know whether lysosomal escape was indeed improved by their design. This study highlights some of the complex biology that underlies successful delivery of a cytotoxic payload to its site of action within a target cell. Identifying critical steps in that process and seeking ways to improve upon each step is how the field will achieve the promise of ADC technology.

\subsection{Importance of Payload Placement}

The advantages afforded by site-specific as compared to heterogeneous conjugation in terms of improved ADC biophysical characteristics and facile analytics are now well-established and have been reviewed elsewhere [10]. While not all site-specific technologies offer flexibility with respect to payload placement, a consensus is growing in the field that the conjugation site can affect ADC biophysical and functional outcomes [21, 52-54]. Two recent studies provide striking examples of this reality. The most surprising is a paper from Pfizer that described the development of a new spliceostatin payload, thailanstatin A [55]. Based on initial data obtained using heterogeneous conjugation through native lysine or cysteine residues, this payload appeared to require the use of lysine conjugation for optimal ADC potency. However, further work using site-specific conjugation at a range of locations revealed that the activity of the payload is highly dependent on conjugation site and that the native hinge cysteine residues accessed for heterogeneous cysteine conjugation made poor placement sites for this payload. The authors did not yet have a mechanistic explanation for this phenomenon but stated that such studies were underway. Another paper described a more well understood effect of site-specific payload placement, namely that conjugation at certain positions can improve linker stability, presumably by shielding the linker from access to enzymatic activity (e.g., proteases, esterases). Dorywalska et al. [56] provided a nice study showing that an enzyme in mouse serum (carboxylesterase 1C [Ces1C]) can cleave ValCit-containing linkers. Then, the authors increased serum stability through two approaches-either by making the linker a worse Ces1C substrate through modifications to the $\mathrm{P} 3$ position or by protecting the linker from Ces $1 \mathrm{C}$ access (presumably) through judicious payload placement at specific locations on the antibody. Both of these studies demonstrated the utility of site-specific payload placement for performing SAR studies at the intact conjugate level in order to fine tune ADC biophysical and functional properties.

\section{Looking Forward in ADC Development}

The ADC field is in a good space. Yes, it has been humbled by clinical failures. But now, after more than 40 years, the field is finally armed with access to all of the elements required for success-including fully human/humanized monoclonal antibodies, site-specific conjugation approaches, a range of potent cytotoxic payloads with various mechanisms of action, versatile linker technologies, and sophisticated analytics. As described in this review, researchers will continue to investigate the sources of poor efficacy or off-target toxicity and will find ways to improve the therapeutic index based on their findings. This will likely be an iterative process, but the progress will be meaningful and cumulative. Meanwhile, some ADCs currently in later stages of the clinical pipeline have shown promising results and may lead to more near-term approvals in this class. ADCs to watch include rovalpituzumab tesirine (targeting DLL3) [26], mirvetuximab soravtansine (targeting folate receptor alpha) [57], glembatumumab vedotin (targeting glycoprotein NMB)[58], and sacituzumab govitecan (targeting TROP-2) [59].

In terms of novel entries to the ADC space, look for an expansion of therapeutic indications beyond oncology. Opportunities for improved therapeutics made through bioconjugation exist in infectious disease, where an antibody-antibiotic conjugate was shown to be more effective than the free antibiotic payload for treating infections caused by drug-resistant bacteria [60]. ADCs and related conjugates can also help to improve treatment of chronic conditions-e.g., autoimmune [61] and cardiovascular diseases-through reducing side effects by selective payload delivery. For example, Lim et al. [62] used an antibody conjugate to selectively deliver a small-molecule agonist against liver $\mathrm{X}$ receptor (LXR) to monocytes/macrophages for treatment of atherosclerosis. The concept represents an advance over dosing with the free payload, because the drug has negative effects on the liver, which also expresses the LXR protein [62].

Finally, for a potential paradigm shift in ADC development, look towards technologies that aim to achieve targeted drug delivery in the absence of an internalizing antigen. Two approaches toward this end have been described. One involves the use of (cytotoxic) payloads that can induce cell death by mediating signals at the cell surface [63]. The other involves a two-step drug-delivery method whereby the targeting and delivery steps are functionally and temporally uncoupled such that an antibody against a non-internalizing target antigen could deliver the payload to the cell surface (step 1) to be followed by payload release induced by a systemically-delivered small molecule (step 2). Rossin et al. [64] described 
this innovative concept as "click to release" chemistry. It employs a bioorthogonal inverse-electron-demand DielsAlder pyridazine elimination reaction to induce self-immolative payload release. One could envision that this approach might someday be used to control timing of the payload delivery, e.g., by dosing the small-molecule release trigger after a washout step to clear systemic conjugates, thereby achieving additional targeting selectivity. Such is the nature of translational medicine that the best creative solutions will find their way into the clinic. The rapid changes in the ADC field will continue in the nearterm, and it will be exciting to watch as new means of making improved therapeutics emerge and take shape from preclinical ideas.

\section{Compliance with Ethical Standards}

Conflicts of interest Penelope Drake and David Rabuka are employees of Catalent Biologics. They have no other conflicts of interest to declare.

Funding No additional funding was received for this work.

Open Access This article is distributed under the terms of the Creative Commons Attribution-NonCommercial 4.0 International License (http://creativecommons.org/licenses/by-nc/4.0/), which permits any noncommercial use, distribution, and reproduction in any medium, provided you give appropriate credit to the original author(s) and the source, provide a link to the Creative Commons license, and indicate if changes were made.

\section{References}

1. Strebhardt K, Ullrich A. Paul Ehrlich's magic bullet concept: 100 years of progress. Nat Rev Cancer. 2008;8:473-80.

2. Drake PM, Rabuka D. An emerging playbook for antibody. Curr Opin Chem Biol. 2015;28:174-80.

3. Mould DR, Meibohm B. Drug development of therapeutic monoclonal antibodies. BioDrugs. 2016;30:275-93.

4. Govindan SV, Sharkey RM, Goldenberg DM. Prospects and progress of antibody-drug conjugates in solid tumor therapies. Expert Opin Biol Ther. 2016;16:883-93.

5. Shor B, Gerber H-P, Sapra P. Preclinical and clinical development of inotuzumab-ozogamicin in hematological malignancies. Mol Immunol. 2015;67:107-16.

6. Lamb YN. Inotuzumab ozogamicin: first global approval. Drugs. 2017;77:1603-10.

7. Beck A, Goetsch L, Dumontet C, Corvaia N. Strategies and challenges for the next generation of antibody-drug conjugates. Nat Rev Drug Discov. 2017;16:315-37.

8. Seattle Genetics stops all trials of ' 33 A blood cancer drug after patient deaths. In: FierceBiotech. 2017. http://www.fiercebiotech. $\mathrm{com} / \mathrm{biotech} / \mathrm{seattle-genetics-stops-all-trials-33a-blood-cancer-}$ drug-after-patient-deaths. Accessed 25 Oct 2017.

9. Seattle Genetics Highlights Phase 1 Vadastuximab Talirine (SGN-CD33A; 33A) Data Presentations, Including Combination Therapy with HMAs, in Patients with Acute Myeloid Leukemia at ASH Annual Meeting. In: investor.seattlegenetics.com. 2016. http://investor.seattlegenetics.com/phoenix.zhtml?c=124860\&p= irol-newsArticle\&ID=2227547. Accessed 25 Oct 2017.
10. Polakis P. Antibody drug conjugates for cancer therapy. Pharmacol Rev. 2015;68:3-19.

11. Saber H, Leighton JK. An FDA oncology analysis of antibodydrug conjugates. Regul Toxicol Pharmacol. 2015;71:444-52.

12. de Goeij BE, Lambert JM. New developments for antibody-drug conjugate-based therapeutic approaches. Curr Opin Immunol. 2016;40:14-23.

13. Doronina SO, Toki BE, Torgov MY, Mendelsohn BA, Cerveny CG, Chace DF, et al. Development of potent monoclonal antibody auristatin conjugates for cancer therapy. Nat Biotechnol. 2003;21:778-84.

14. Dubowchik GM, Firestone RA, Padilla L, Willner D, Hofstead SJ, Mosure K, et al. Cathepsin B-labile dipeptide linkers for lysosomal release of doxorubicin from internalizing immunoconjugates: model studies of enzymatic drug release and antigenspecific in vitro anticancer activity. Bioconjug Chem. 2002;13:855-69.

15. Zhao H, Gulesserian S, Malinao MC, Kumar-Ganesan S, Song J, Chang MS, et al. A potential mechanism for ADC-induced neutropenia: role of neutrophils in their own demise. Mol Cancer Ther. 2017. https://doi.org/10.1158/1535-7163.MCT-17-0133.

16. Uppal H, Doudement E, Mahapatra K, Darbonne WC, Bumbaca $\mathrm{D}$, Shen BQ, et al. Potential mechanisms for thrombocytopenia development with trastuzumab emtansine (T-DM1). Clin Cancer Res. 2015;21:123-33.

17. Poon KA, Flagella K, Beyer J, Tibbitts J, Kaur S, Saad O, et al. Preclinical safety profile of trastuzumab emtansine (T-DM1): mechanism of action of its cytotoxic component retained with improved tolerability. Toxicol Appl Pharmacol. 2013;273:298-313.

18. Sun X, Ponte JF, Yoder NC, Laleau R, Coccia J, Lanieri L, et al. Effects of drug-antibody ratio on pharmacokinetics, biodistribution, efficacy, and tolerability of antibody-maytansinoid conjugates. Bioconjug Chem. 2017;28:1371-81.

19. Lyon RP, Bovee TD, Doronina SO, Burke PJ, Hunter JH, NeffLaFord HD, et al. Reducing hydrophobicity of homogeneous antibody-drug conjugates improves pharmacokinetics and therapeutic index. Nat Biotechnol. 2015;33:733-5.

20. Alley SC, Benjamin DR, Jeffrey SC, Okeley NM, Meyer DL, Sanderson RJ, et al. Contribution of linker stability to the activities of anticancer immunoconjugates. Bioconjug Chem. 2008; 19:759-65.

21. Shen B-Q, Xu K, Liu L, Raab H, Bhakta S, Kenrick M, et al. Conjugation site modulates the in vivo stability and therapeutic activity of antibody-drug conjugates. Nat Biotechnol. 2012;30:184-9.

22. Rago B, Tumey LN, Wei C, Barletta F, Clark T, Hansel S, et al. Quantitative conjugated payload measurement using enzymatic release of antibody-drug conjugate with cleavable linker. Bioconjug Chem. 2017;28:620-6.

23. Lyon RP, Setter JR, Bovee TD, Doronina SO, Hunter JH, Anderson ME, et al. Self-hydrolyzing maleimides improve the stability and pharmacological properties of antibody-drug conjugates. Nat Biotechnol. 2014;32:1059-62.

24. Tumey LN, Charati M, He T, Sousa E, Ma D, Han X, et al. Mild method for succinimide hydrolysis on ADCs: impact on ADC potency, stability, exposure, and efficacy. Bioconjug Chem. 2014;25:1871-80.

25. Stein AS, Walter RB, Erba HP, Fathi AT, Advani AS, Lancet JE, et al. A phase 1 trial of SGN-CD33A as monotherapy in patients with CD33-positive acute myeloid leukemia (AML). Blood. 2015;126:324.

26. Rudin CM, Pietanza MC, Bauer TM, Ready N, Morgensztern D, Glisson BS, et al. Rovalpituzumab tesirine, a DLL3-targeted antibody-drug conjugate, in recurrent small-cell lung cancer:a 
first-in-human, first-in-class, open-label, phase 1 study. Lancet Oncol. 2016;18:42-51.

27. Bross PF, Beitz J, Chen G, Chen XH, Duffy E, Kieffer L, et al. Approval summary: gemtuzumab ozogamicin in relapsed acute myeloid leukemia. Clin Cancer Res. 2001;7:1490-6.

28. Ma Y, Khojasteh SC, Hop CECA, Erickson HK, Polson A, Pillow $\mathrm{TH}$, et al. Antibody drug conjugates differentiate uptake and DNA alkylation of pyrrolobenzodiazepines in tumors from organs of xenograft mice. Drug Metab Dispos. 2016;44:1958-62.

29. Hinrichs MJM, Ryan PM, Zheng B, Afif-Rider S, Yu XQ, Gunsior M, et al. fractionated dosing improves preclinical therapeutic index of pyrrolobenzodiazepine-containing antibody drug conjugates. Clin Cancer Res. 2017. https://doi.org/10.1158/10780432.CCR-17-0219.

30. Cilliers C, Guo H, Liao J, Christodolu N, Thurber GM. Multiscale modeling of antibody-drug conjugates: connecting tissue and cellular distribution to whole animal pharmacokinetics and potential implications for efficacy. AAPS J. 2016. https://doi.org/ 10.1208/s12248-016-9940-z.

31. Gerber H-P, Sapra P, Loganzo F, May C. Combining antibodydrug conjugates and immune-mediated cancer therapy: what to expect? Biochem Pharmacol. 2016;102:1-6.

32. Muller P, Martin K, Theurich S, Schreiner J, Savic S, Terszowski $\mathrm{G}$, et al. Microtubule-depolymerizing agents used in antibodydrug conjugates induce antitumor immunity by stimulation of dendritic cells. Cancer Immunol Res. 2014;2:741-55.

33. Martin K, Müller P, Schreiner J, Prince SS, Lardinois D, Heinzelmann-Schwarz VA, et al. The microtubule-depolymerizing agent ansamitocin P3 programs dendritic cells toward enhanced anti-tumor immunity. Cancer Immunol Immunother. 2014;63:925-38.

34. Müller P, Kreuzaler M, Khan T, Thommen DS, Martin K, Glatz $\mathrm{K}$, et al. Trastuzumab emtansine (T-DM1) renders HER2+ breast cancer highly susceptible to CTLA-4/PD-1 blockade. Sci Transl Med. 2015;7:315ra188.

35. Li F, Ulrich M, Jonas M, Stone IJ, Linares G, Zhang X, et al. Tumor-Associated macrophages can contribute to antitumor activity through Fc $\gamma \mathrm{R}$-mediated processing of antibody-drug conjugates. Mol Cancer Ther. 2017;16:1347-54.

36. Pfeifer M, Zheng B, Erdmann T, Koeppen H, McCord R, Grau $\mathrm{M}$, et al. Anti-CD22 and anti-CD79B antibody drug conjugates are active in different molecular diffuse large B-cell lymphoma subtypes. Leukemia. 2015;29:1578-86.

37. Bartlett NL, Smith MR, Siddiqi T, Advani RH, O'Connor OA, Sharman JP, et al. Brentuximab vedotin activity in diffuse large B-cell lymphoma with CD30 undetectable by visual assessment of conventional immunohistochemistry. Leuk Lymphoma. 2017;58:1607-16.

38. Jacobsen ED, Sharman JP, Oki Y, Advani RH, Winter JN, Bello $\mathrm{CM}$, et al. Brentuximab vedotin demonstrates objective responses in a phase 2 study of relapsed/refractory DLBCL with variable CD30 expression. Blood. 2015;125:1394-402.

39. Chang C-H, Wang Y, Zalath M, Liu D, Cardillo TM, Goldenberg DM. Combining ABCG2 inhibitors with IMMU-132, an AntiTrop-2 antibody conjugate of $\mathrm{SN}-38$, overcomes resistance to SN-38 in breast and gastric cancers. Mol Cancer Ther. 2016;15:1910-9.

40. Cardillo TM, Sharkey RM, Rossi DL, Arrojo R, Mostafa AA, Goldenberg DM. Synthetic lethality exploitation by an AntiTrop-2-SN-38 antibody-drug conjugate, IMMU-132, plus PARP inhibitors in BRCA1/2-wild-type triple-negative breast cancer. Clin Cancer Res. 2017;23:3405-15.

41. Damelin M, Bankovich A, Bernstein J, Lucas J, Chen L, Williams S, et al. A PTK7-targeted antibody-drug conjugate reduces tumor-initiating cells and induces sustained tumor regressions.
Sci Transl Med. 2017. https://doi.org/10.1126/scitranslmed. aag2611.

42. Seaman S, Zhu Z, Saha S, Zhang XM, Yang MY, Hilton MB, et al. Eradication of tumors through simultaneous ablation of CD276/B7-H3-positive tumor cells and tumor vasculature. Cancer Cell. 2017;31:501-8.

43. Polson AG, Calemine-Fenaux J, Chan P, Chang W, Christensen E, Clark S, et al. Antibody-drug conjugates for the treatment of non-Hodgkin's lymphoma: target and linker-drug selection. Cancer Res. 2009;69:2358-64.

44. Ritchie M, Tchistiakova L, Scott N. Implications of receptormediated endocytosis and intracellular trafficking dynamics in the development of antibody drug conjugates. mAbs. 2014;5:13-21.

45. DeVay RM, Delaria K, Zhu G, Holz C, Foletti D, Sutton J, et al. Improved lysosomal trafficking can modulate the potency of antibody drug conjugates. Bioconjug Chem. 2017;28:1102-14.

46. de Goeij BECG, Satijn D, Freitag CM, Wubbolts R, Bleeker WK, Khasanov A, et al. High turnover of tissue factor enables efficient intracellular delivery of antibody-drug conjugates. Mol Cancer Ther. 2015;14:1130-40.

47. Staben LR, Koenig SG, Lehar SM, Vandlen R, Zhang D, Chuh J, et al. Targeted drug delivery through the traceless release of tertiary and heteroaryl amines from antibody-drug conjugates. Nat Chem. 2016;8:1112-9.

48. Kolakowski RV, Haelsig KT, Emmerton KK, Leiske CI, Miyamoto JB, Cochran JH, et al. The methylene alkoxy carbamate self-immolative unit: utilization for the targeted delivery of alcohol-containing payloads with antibody-drug conjugates. Angew Chem Int Ed. 2016;55:7948-51.

49. Zhang D, Pillow TH, Ma Y, Cruz-Chuh JD, Kozak KR, Sadowsky JD, et al. Linker immolation determines cell killing activity of disulfide-linked pyrrolobenzodiazepine antibody-drug conjugates. ACS Med Chem Lett. 2016;7:988-93.

50. Zhang D, Yu S-F, Ma Y, Xu K, Dragovich PS, Pillow TH, et al. Chemical structure and concentration of intratumor catabolites determine efficacy of antibody drug conjugates. Drug Metab Dispos. 2016;44:1517-23.

51. Singh R, Setiady YY, Ponte J, Kovtun YV, Lai KC, Hong EE, et al. A new triglycyl peptide linker for antibody-drug conjugates (ADCs) with improved targeted killing of cancer cells. Mol Cancer Ther. 2016;15:1311-20.

52. Strop P, Liu S-H, Dorywalska M, Delaria K, Dushin RG, Tran T-T, et al. Location matters: site of conjugation modulates stability and pharmacokinetics of antibody drug conjugates. Chem Biol. 2013;20:161-7.

53. Drake PM, Albers AE, Baker J, Banas S, Barfield RM, Bhat AS, et al. Aldehyde tag coupled with HIPS chemistry enables the production of ADCs conjugated site-specifically to different antibody regions with distinct in vivo efficacy and PK outcomes. Bioconjug Chem. 2014;25:1331-41.

54. Strop P, Delaria K, Foletti D, Witt JM, Hasa-Moreno A, Poulsen $\mathrm{K}$, et al. Site-specific conjugation improves therapeutic index of antibody drug conjugates with high drug loading. Nat Biotechnol. 2015;33:694-6.

55. Puthenveetil S, He H, Loganzo F, Musto S, Teske J, Green M, et al. Multivalent peptidic linker enables identification of preferred sites of conjugation for a potent thialanstatin antibody drug conjugate. PLoS One. 2017;12:e0178452.

56. Dorywalska M, Dushin R, Moine L, Farias SE, Zhou D, Navaratnam T, et al. Molecular basis of valine-citrulline-PABC linker instability in site-specific ADCs and its mitigation by linker design. Mol Cancer Ther. 2016;15:958-70.

57. Moore KN, Martin LP, Matulonis UA, Konner JA. IMGN853 (mirvetuximab soravtansine), a folate receptor alpha (FR $\alpha)$ - 
targeting antibody-drug conjugate (ADC): single-agent activity in platinum-resistant. J Clin Oncol. 2016;35:15.

58. Yardley DA, Weaver R, Melisko ME, Saleh MN, Arena FP, Forero A, et al. EMERGE: a randomized phase II study of the antibody-drug conjugate glembatumumab vedotin in advanced glycoprotein NMB-expressing breast cancer. J Clin Oncol. 2015;33:1609-19.

59. Burki TK. Sacituzumab govitecan activity in advanced breast cancer. Lancet Oncol. 2017;18:e246.

60. Lehar SM, Pillow T, Xu M, Staben L, Kajihara KK, Vandlen R, et al. Novel antibody-antibiotic conjugate eliminates intracellular S. aureus. Nature. 2015;527:323-8.

61. Kern JC, Cancilla M, Dooney D, Kwasnjuk K, Zhang R, Beaumont $\mathrm{M}$, et al. Discovery of pyrophosphate diesters as tunable, soluble, and bioorthogonal linkers for site-specific antibody-drug conjugates. J Am Chem Soc. 2016;138:1430-45.

62. Lim RKV, Yu S, Cheng B, Li S, Kim N-J, Cao Y, et al. Targeted delivery of LXR agonist using a site-specific antibody-drug conjugate. Bioconjug Chem. 2015;26:2216-22.

63. Marshall DJ, Harried SS, Murphy JL, Hall CA, Shekhani MS, Pain C, et al. Extracellular antibody drug conjugates exploiting the proximity of two proteins. Mol Ther. 2016;24:1760-70.

64. Rossin R, van Duijnhoven SMJ, ten Hoeve W, Janssen HM, Kleijn LHJ, Hoeben FJM, et al. Triggered drug release from an antibody-drug conjugate using fast "click-to-release" chemistry in mice. Bioconjug Chem. 2016;27:1697-706. 